2. Treatment of acute suppurative otitis media by a sulphonamide should be reserved for the period after incision of the membrane and bacteriological investigation. If the fever is maintained, the appropriate sulphonamide may be used in full dosage (day and night), but not after the end of the second week.

3. After two, three, or four weeks the condition of the ear, if still actively suppurating, is again becoming one that potentially requires surgical drainage, this time of the mastoid; and signs and symptoms justifying intervention must not be masked by the administration of sulphonamides, which at best will rarely reach the local area but will distort the clinical picture as a whole.

4. Wholesale use of sulphonamides in otitic infections is to be deprecated.

5. If a sulphonamide has been given before drainage, the most valuable help in assessing the beneficial effect of the drug is gained by examining the drum-head frequently. If this continues to bulge myringotomy should not be delayed, because the condition, which otherwise appears to be improving, is in reality becoming more perilous. Persistence of deafness is also a strong indication that all is not well.

6. Patients taking sulphonamide drugs sometimes do not acquire any resistance of their own to the infection with which they are contending, and it would seem that recurrences are more likely in these cases, which, in addition to developing no immunity to the infection, may find the micro-organism has become sulphonamideresistant. Recently a schoolboy had five extremely severe and prolonged attacks of tonsillitis (haemolytic streptococci) over a period of three and a half months; each attack lasted at least a fortnight, with high fever. All attacks, except the last, were treated with sulphonamides, and it was assumed that, in addition to developing no individual immunity, the micro-organism had become sulphonamide-resistant. Tonsillectomy was uneventful, and has resulted in freedom from any further trouble.

7. It is needless to emphasize the uselessness and danger of giving inadequate or erratic dosage of sulphonamides. If given at all, full doses at four-hourly intervals, day and night, are required for the full period, and the drug should be continued for a time after complete subsidence of fever.

\section{General Comment}

It would seem, then, that the chief dangers to be met with in cases of otitis treated with sulphonamides are found in those in which this has been the sole treatment. Such cases appear to the unwary to have been completely relieved, and no further anxiety is felt about the ear condition-and there may be much to support such an attitude. Sometimes the middle ear may return to normal, with recovery of hearing; more often the tympanic membrane, still intact, will remain oedematous, the middle ear congested, and with much loss of hearing. Mastoid tenderness is frequently absent. In such a case it is difficult to know what has been happening in the three or four weeks following the initial infection and apparent subsidence, but such signs and symptoms as pallor, loss of weight, dirty tongue, vague headaches, and (probably most important of all) congestion of the unperforated drum-head, with deafness, should give rise to the suspicion that a slow, latent, and insidious infective process which will not undergo resolution is going on all the time. Such masking is the origin of greater potential dangers than those seen in pre-sulphonamide days, in which the course of the infective process could be clearly followed.

Some help can be gained by radiography, and this should always be used in doubtful cases. Mastoid tenderness and swelling in these atypical cases usually manifest themselves late, about the fifth or sixth week, but not before a critical latent phase has passed, in which the lateral sinus, the meninges, or the brain might just as easily have provided the first sites for an extension of the original infection.

In cases, or suspected cases, of thrombophlebitis of the lateral sinus and brain abscess, reliance should not be placed on sulphonamide drugs, which (a) will fail to eliminate the infection and so lead to catastrophe, and $(b)$ will mask the indications which would suggest the necessity for surgical intervention.

One must remember that sulphonamides bring about a condition of bacteriostasis ; unless the patient's immunity is sufficiently raised, either artificially by appropriate serum or naturally by his own efforts, the micro-organism will be capable of regaining its activity. This was well seen in Case 2, in which large doses of sulphadiazine $(60 \mathrm{~g}$. in 8 days) brought about such a great improvement that the hemiparesis and hemianaesthesia were almost eliminated and the signs of meningitis had practically cleared; within a few hours of stopping the drug, however, the patient relapsed into coma, with turbid C.S.F. and incontinence, and was only restored to consciousness with the greatest difficulty by means of intravenous sulphapyridine. Large doses of intravenous serum and penicillin intrathecally and intramuscularly led to eventual recovery. It would appear possible that, in the future, it will be proved that penicillin is capable of bringing about sterilization and absorption of purulent foci, and so dispense with the need for surgical drainage in these cases; this is a feat which it is beyond the power of sulphonamides to achieve.

\section{BONE-MARROW TRANSFUSION IN INFANTS AND CHILDREN}

\section{INTRODUCING A SPECIALLY DESIGNED NEEDLE}

\author{
BY
}

JANET D. GIMSON, M.B., B.S., D.C.H.

Resident Assistant Physician, the Hospital for Sick Children, Great Ormond Street

Some skill and experience are required to insert a needle or cannula by the cut-down technique into a vein of the ankle, cubital fossa, wrist, or scalp of premature and small infants. These, however, are a group of patients who not infrequently need parenteral fluid. An attempt has therefore been made to develop a more simple method than the intravenous, and one which would be at least as safe and sure. The bone marrow of the tibia was the site elected. The marrow cavity in the sternum of infants, and in fact of considerably older children, is not large enough for this to be a practicable place for the reception of transfusion agents. Hamilton Bailey (1944) has reviewed the present position with regard to bone marrow for this purpose, and is favourably impressed. He mentions the winged Witts sternal-puncture needle as admirable for cannulization of the sternum in adults. In my opinion, however, a Witts needle or a Tocantins needle leaves much to be desired in use for an infant.
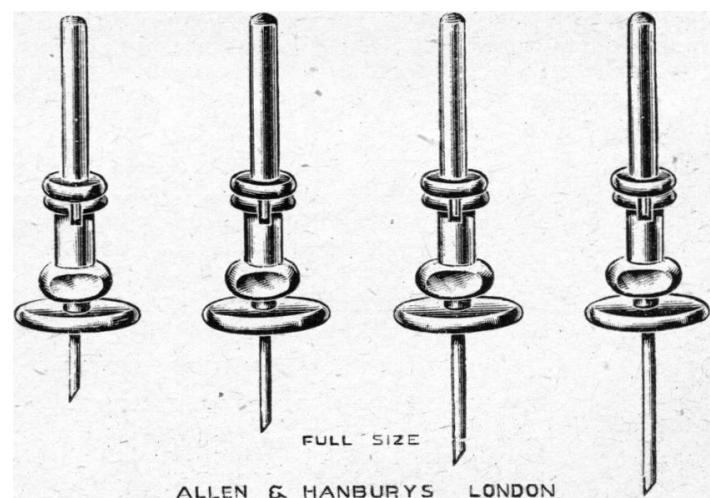

ALILEN \& HANBURY
Fig. 1
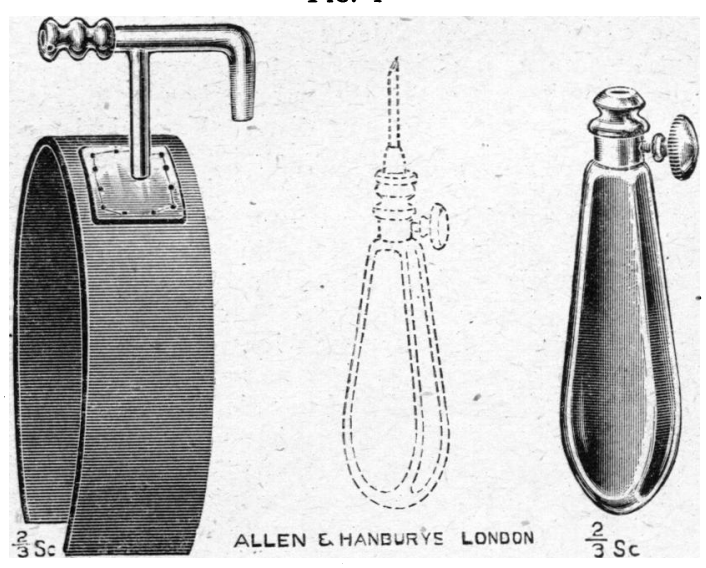

Fig. 2

The needle illustrated (Figs. 1 and 2) was designed for transfusions into the tibial marrow of infants and children. The following features may be noted: 
(a) The needle is light.

(b) The distance between the mount and the flange is short.

(c) The flange is immovable, and so the extra weight of a screw mechanism is avoided. The least possible movement of the needle in the cortical bone is thus allowed and this junction remains firm.

(d) Four different lengths of needle below the flange have been made : $1 / 4$ in., $3 / 8$ in., $1 / 2$ in.; and 5/8 in. Size 18 s.w.G. is suggested for the two smaller; size 16 or 17 s.w.G. for the larger. The 1/4-in. size is suitable for premature infants and neonates; the $1 / 2$-in. and 5/8-in. for about 5-year-old children or over. But the length of needle used should be according to the size of the child and the amount of subcutaneous fat.

(e) Each needle has its own stylet.

(f) There is one handle for the set, which can be screwed on to the stylet in order to get a good grip during insertion of the needle.

(g) The right-angled adaptor, with the strut to keep it firmly in position, leads the tubing along parallel to the limb. The rubber strip attached to the base of the strut is strapped round the limb Occasionally, owing to fat or oedema or some other irregularity of contour, the strut on the adaptor seems too long and will not sit on the leg and in the needle satisfactorily. An adaptor without a strut is therefore supplied in each set. The tubing should be fixed in two places with strapping-to the limb and to the splint; the splint should extend 4 in. to 6 in. beyond the foot.

\section{Technique of Insertion}

The place chosen on the tibia is the flat subcutaneous plate of bone inferior and medial to the anterior tibial tuberosity. This area is well below the epiphysial line, above the nutrient artery, and there are no important anatomical structures to which damage might be done between the skin and bone. It will be remembered that a cross-section of the tibia is roughly triangular. A non-touch technique is maintained so far as is possible, as during any operation involving bone.

1. The limb is splinted in a semi-externally rotated position (as for cutting down on to the internal saphenous vein at the ankle).

2. The skin is cleaned. Local anaesthesia of the skin, subcutaneous tissue, and periosteum is given with $1 \%$ novocain. The area is massaged to disperse the anaesthetic, and the anterior and medial borders of the tibia are palpated.

3. The needle and stylet, with the handle attached, are taken and, with the needle at right angles to the plate of bone-i.e., pointing postero-laterally with reference to the leg itself-are inserted with a firm twisting motion as when using a bradawl. The needle passes through the cortical bone with a crisp crunch. The flange rests lightly on the skin.

4. When the stylet is withdrawn a small amount of marrow may well up or be sucked up into a syringe; it seldom flows out freely.

5. The adaptor, filled with the transfusion fluid and attached to the tubing from the dripper and bottle (which has already had air bubbles excluded), is fitted to the needle and the flow started.

Fig. 3 shows the arrangement for transfusion through the bone marrow of the tibia.

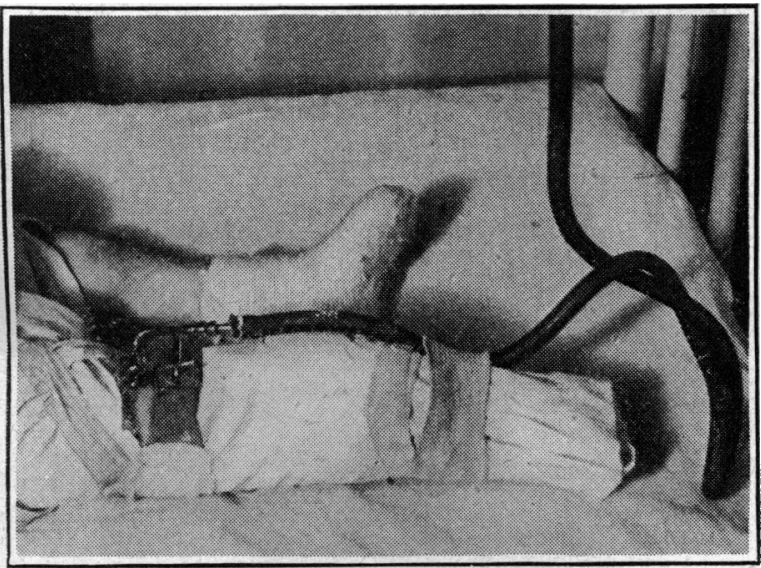

Fig. 3.-Close-up of the arrangement for transfusing through the tibia in the case of a 3-months-old boy with bilateral mastoiditis.

Any of the usual transfusion fluids may be given in this way-saline, Hartmann's solution, blood, serum, or plasma. When using blood it is often necessary to increase the pressure by raising the bottle as much as six feet above the needle ; or, alternatively, the blood may be diluted. The constant rate of drip which can be maintained is usually remarkable; the rate required is adjustable by a screw clip on the tubing. The older the child the larger the marrow cavity and therefore the faster the flow can be. The longest time one transfusion has been maintained was six days. When the needle was taken out it was still firmly held in the bone, and there was no reason to suppose that the transfusion could not have been continued had it been necessary.

Particular attention might be paid to the following points:

Emphasis is laid on the necessity for inserting the needle at right angles to the surface of the tibia. This is facilitated by the position of semi-external rotation of the limb. The diagram (Fig. 4) shows the error of attempting to insert the
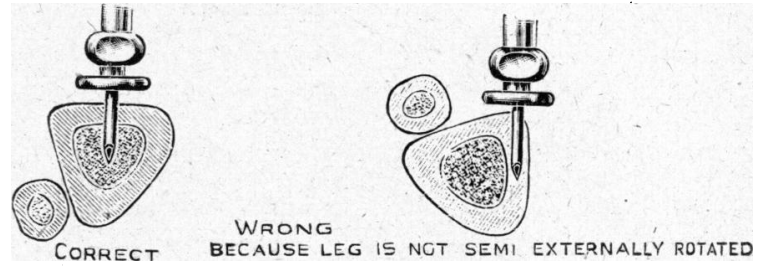

Fig. 4

needle in the antero-posterior direction of the limb, as might happen if the leg were not fixed in the correct position. It is probably wise to inject a small amount of saline through the needle as soon as it has been inserted, to ensure that the marrow has been entered satisfactorily. The adaptor can then be securely connected.

If the needle does work loose, fluid will leak back into the subcutaneous tissues and the limb will become oedematous; the transfusion must then be stopped. A careful watch for oedema should be kept during the first hour or two of the transfusion, because, if oedema is allowed to develop, a pressure necrosis of the skin beneath the flange might result.

Dislodging by kicking with the other leg can be prevented by anchoring it with a bandage noose round the ankle. A small cradle will prevent disturbance by the bed-clothes.

If a second transfusion is required soon after the first has been discontinued the other tibia must of course be employed, as leakage back through the original hole will make that leg impracticable.

It would seem wise to reiterate the need for strict asepsis, since osteomyelitis, though it remains theoretical, is the haunting danger.

The procedure can be facilitated by giving a feed or an appropriate dose of chloral hydrate; these will quiet the baby and often surprisingly help the initial stages of the transfusion.

I want to thank Dr. Donald Paterson and Mr. T. Twistington Higgins for their interest, and $\mathrm{Dr}$. King Lewis for his encouragement and suggestions. The needle and attachments have been made by Messrs. Allen and Hanburys Ltd.

$$
\text { REFERENCE }
$$

Bailey, Hamilton (1944), British Medical Journal, 1, 181

\section{“CELlOPHANE" FOR THE TREATMENT OF BURNS}

BY

JOHN FARR, M.D., F.R.C.S.Ed.

Major, R.A.M.C.

Since Davidson's communication in 1925 many other observers have contributed to the literature on the treatment of burns. Usually their methods have had at least two factors in common: first, the exclusion of air, to promote the patient's comfort and to diminish shock and infection; secondly, to allow epithelization to proceed undisturbed by frequent and painful dressings. Within the last three years some observers have emphasized the similarity between the pathology of a burn and that of other wounds, and have reiterated the harm of tissue irritants in the toilet of the burn and demonstrated the value of firm pressure upon the area to support the damaged vascular bed. In the American literature the application of these principles has come to be known as the "cleansing, rest, and compression" treatment of burns. The 